Original Article

\title{
SYNTHESIS, CHARACTERIZATION, AND BIOLOGICAL ACTIVITY OF NOVEL N- PHENYLPROPYL-3-SUBSTITUTED INDOLINE-2-ONE DERIVATIVES
}

\author{
PUSHPA ${ }^{1,2}$, BASAVARAJ S. NARABOLI ${ }^{1}$, J. S. BIRADAR ${ }^{1^{*}}$ \\ 1Department of Studies and Research in Chemistry, Gulbarga University, Kalaburagi 585106, Karnataka, India, ${ }^{2}$ Government First, Grade \\ College, Raichur, Karnataka, India \\ Email: jsbiradar@rediffmail.com
}

Received: 02 Jul 2017 Revised and Accepted: 31 Aug 2017

\section{ABSTRACT}

Objective: The objective of the present work deals with the synthesis, characterization and evaluation of antimicrobial and antioxidant activity of $N$-phenylpropyl-3-substituted indoline-2-one derivatives.

Methods: A series of new 3-hydroxy-3-(2-oxoethyl)-1-(3-phenylpropyl) indolin-2-one derivatives 3(a-l) and 3-(2-oxoethylidene)-1-(3phenylpropyl) indolin-2-one derivatives 4(a-l) were synthesized by knoevenagel condensation of $N$-phenylpropyl-5-substituted indole-2,3-diones with various acetophenones analogues. The chemical structures of synthesized compounds were confirmed by IR, ${ }^{1} \mathrm{HNMR}$ and Mass spectroscopic and elemental data. These compounds were also screened for their in vitro antimicrobial and antioxidant activities.

Results: Novel compounds 3-hydroxy-3-(2-oxoethyl)-1-(3-phenylpropyl) indolin-2-one derivatives 3(a-l) and 3-(2-oxoethylidene)-1-(3phenylpropyl) indolin-2-one derivatives $4(\mathrm{a}-\mathrm{l})$ were synthesised and characterized using spectral and analytical data. The results of antibacterial and antifungal and antioxidant activities showed that some of the synthesized compounds exhibited promising results.

Conclusion: All the newly synthesized compounds were screened for antimicrobial activity by cup plate method and antioxidant activity by the DPPH method using Ciprofloxacin and Amphotericin B as standards against gram positive and gram negative bacteria and fungi respectively.

Keywords: Indole-2,3-diones, Pyridine, Thiophene, Antimicrobial, Antioxidant

(C) 2017 The Authors. Published by Innovare Academic Sciences Pvt Ltd. This is an open access article under the CC BY license (http://creativecommons.org/licenses/by/4.0/) DOI: http://dx.doi.org/10.22159/ijpps.2017v9i10.21083

\section{INTRODUCTION}

Infectious diseases are the main cause of mortality in the world and rapid increase of antimicrobial resistance among pathogenic strains (bacterial and fungal) is becoming a serious public health problem because microbes replicate very rapidly and get mutated which help the microbes to survive in the presence of an antimicrobial drug, these will quickly become predominant throughout the microbial population.

Free radicals play important roles in many physiological and pathological conditions [1]. In, general, the generation and scavenging of oxygen free radicals is balanced and any imbalance or excessive amounts of active radicals may contribute to disease development. It has been found that free radical reactions can produce deleterious modifications in membranes, proteins, enzymes, and DNA [2], increasing the risk of diseases such as cancer [3], Alzheimer's [4], Parkinson's [5], angiocardiopathy [6], arthritis [7], asthma [8], diabetes [9] and degenerative eye disease [10].

Owing to increased microbial resistance, and various disorders caused by free radicals, and to develop more potent small molecules with enhanced antimicrobial and antioxidant properties, a series of $\mathrm{N}$-phenylpropyl-3-substituted indoline-2-ones are synthesized by Knovenegal condensation.

Indole-1H-2, 3-dione or Indoline-2, 3-dione commonly known as Isatin is a well-known natural product found in plants of genus Isatis and in couropita guianancis aubl [11]. It has also been isolated as a metabolic derivative of adrenaline in humans [12]. The biological and pharmacological properties of isatin and its derivatives have led to extensive use of these compounds as key intermediate in organic synthesis [13]. It is a core constituent of many alkaloids [14], drugs [15], as well as dyes [16]. The literature survey reveals that various derivatives of isatin possess diverse activities such as antibacterial [17], antifungal [18], antiviral [19], HIV [20], antimycobacterial [21], anticancer [22], anti-inflammatory [23], anticonvulsant activities [24] and acts as a potent antagonist on atrial natriuretic peptide receptors in vitro [25].
It possesses an indole nucleus with two chemically distinct cyclic carbonyl groups keto and lactam. The structure of isatin has provoked tremendous interest in chemists to unfold the interesting aspects of organic reactions and mechanism. Isatins mainly react at three different sites, namely aromatic substitution at C-5, Nalkylation and carbonyl reaction at $\mathrm{C}-3$. The most fascinating application of isatins in organic synthesis is undoubtedly due to the highly reactive $\mathrm{C}-3$ carbonyl group that is a prochiral center as well. At the C-3 carbonyl group of isatins, nucleophilic additions or spiro annulation takes place which transforms it into 2-oxoindole derivative. 2-oxoindoles especially those which are spiro fused to the other cyclic framework, have drawn tremendous interest of researchers in the area of synthetic organic chemistry and medicinal chemistry worldwide because they occur in many natural products such as spirotryprostatins, horsfiline, gelsemine, gelseverine, rhynchophylline and elacomine etc.

So as a part of our research in the area of heterocyclic compounds containing indole moiety, the main focus was on $\mathrm{N}$-alkylation and nucleophilic addition at $\mathrm{C}-3$ of isatin with various aromatic and heterocyclic acetophenone analogues. Herein we report the synthesis of some new $\mathrm{N}$-phenylpropyl-3-substituted indoline-2-one derivatives, their characterization and antimicrobial and antioxidant activities.

The reaction of 5-substituted isatin (1) with 3-chloro propyl benzene in the presence of $\mathrm{K} 2 \mathrm{CO} 3$ and $N, N$-dimethyl formamide gave 1-(3phenylpropyl) indoline-2,3-dione (2a) and 5-flouro-1-(3phenylpropyl) indoline-2,3-dione (2b). It was found that the K2CO3DMF system is an effective promotion for this reaction [26]. Use of $\mathrm{K} 2 \mathrm{CO} 3$ as a catalyst has inherent advantages including operational simplicity, low cost and suitability in industrial application. Reaction of 2(a-b) with acetophenone derivatives viz; acetyl naphthalene 2-acetyl thiophene, 3-acetylpyridine, 4-flouro acetophenone, 4-methoxy acetophenone, 4-benzonitrile gave 3-hydroxy-3-(2-oxoethyl)-1-(3phenyl propyl) indoline-2-one derivatives (3a-l). The tertiary alcohol can easily be dehydrated under acidic conditions to yield 3-(2oxoethylidene)-1-(3-phenylpropyl) indolin-2-one derivatives 4(a-1). 


\section{MATERIALS AND METHODS}

\section{Materials}

All the chemicals and solvents were of laboratory reagent grade and used as received from Sigma Aldrich and SD fine. Melting points were determined in open capillaries and are uncorrected. The purity of the compounds was checked by TLC using silica gel-G coated aluminium plates (Merck) and spots were visualized by exposing the dry plates to iodine vapors. The IR (KBr) spectra were recorded on a Perkin-Elmer spectrometer on FT-IR spectrometer. The ${ }^{1} \mathrm{H}$ NMR (DMSO-d6) spectra recorded on a Bruker $(400 \mathrm{MHz})$ and the chemical shifts were expressed in ppm ( $\delta$ scale) downfield from TMS. Mass spectral data were recorded by electron impact method on JEOL GCMATE II GC-MS mass spectrometer. Elemental analysis was carried out using Flash EA 1112 series elemental analyzer. All the compounds gave $\mathrm{C}, \mathrm{H}$ and $\mathrm{N}$ analysis within $\pm 0.5 \%$ of the theoretical values.

General procedure for the synthesis of 5-substituted-1-(3phenylpropyl)indoline-2,3-dione (2a-b)

To a stirred solution of indoline-2,3-dione/5-flouro indoline-2,3dione $(33.9 \mathrm{mmol} / 9 \mathrm{mmol})$ in $\mathrm{N}, \mathrm{N}$-dimethylformamide $(40 \mathrm{ml})$ were added $\mathrm{K}_{2} \mathrm{CO}_{3}(50 \mathrm{mmol})$, 3-chloropropyl benzene $(13.6 \mathrm{mmol})$ and the reaction mixture was stirred at $80{ }^{\circ} \mathrm{C}$ for $16-18 \mathrm{~h}$. Reaction mixture was poured into ice-cold water, precipitated solid was filtered, washed with water, and dried to obtain the desired product as a colorless solid.

\section{1-(3-phenylpropyl)indoline-2,3-dione (2a)}

IR (KBr) ( $\chi_{\max }$ in cm-1): 1604 (NHCO), $1702(\mathrm{C}=0) .{ }^{1} \mathrm{H}$ NMR (400 $\left.\mathrm{MHz}, \mathrm{CDCl}_{3}\right)^{\delta}(\mathrm{ppm}): 2.62\left(\mathrm{t}, 2 \mathrm{H}, \mathrm{CH}_{2}\right), 2.93\left(\mathrm{~m}, 2 \mathrm{H}, \mathrm{CH}_{2}\right), 3.97(\mathrm{t}, 2 \mathrm{H}$, $\mathrm{N}-\mathrm{CH}_{2}$ ), 7.29-7.9 (m, 9H,Ar-H). LCMS: $\mathrm{m} / \mathrm{z}=265$ [M] ${ }^{+}$.

\section{5-fluoro-1-(3-phenylpropyl)indoline-2,3-dione (2b).}

IR (KBr) ( $\chi_{\max }$ in cm-1): 1630 (NHCO), $1710(\mathrm{C}=0) .{ }^{1} \mathrm{H}$ NMR (400 $\left.\mathrm{MHz} \mathrm{CDCl}_{3}\right)^{\delta}(\mathrm{ppm}): 2.62\left(\mathrm{t}, 2 \mathrm{H}, \mathrm{CH}_{2}\right), 2.93\left(\mathrm{~m}, 2 \mathrm{H}, \mathrm{CH}_{2}\right), 3.97(\mathrm{t}, 2 \mathrm{H}$, $\left.\mathrm{N}-\mathrm{CH}_{2}\right), 7.29-7.9(\mathrm{~m}, 8 \mathrm{H}, \mathrm{Ar}-\mathrm{H})$. LCMS: $\mathrm{m} / \mathrm{z}=283[\mathrm{M}]^{+}$.

General procedure for the Synthesis of 3-hydroxy-3-(2oxoethyl)-1-(3-phenylpropyl)indolin-2-one derivatives (3a-l)

To a stirred solution of substituted indole-2,3-dione 1-(3phenylpropyl)-indoline-2,3-dione $(3.75 \mathrm{mmol})$ in ethanol $(20 \mathrm{ml})$ were added piperidine $(11.25 \mathrm{mmol})$, and various acetophenone derivatives like 1-(naphthalen-2-yl)ethan-1-one, 1-(thiophen-2yl)ethan-1-one, 1-(pyridin-3-yl)ethan-1-one, 1-(4-fluorophenyl) ethan-1-one, 1-(4-methoxyphenyl)ethan-1-one (4 mmol), and 4acetylbenzonitrile, the reaction mixture was stirred at room temperature for $6 \mathrm{~h}$. Reaction mixture was filtered, the solid was washed with ethanol, and dried to obtain the product.

3-hydroxy-3-(2-(naphthalen-2-yl)-2-oxoethyl)-1-(3phenylpropyl)indolin-2-one (3a)

IR (KBr) $\left(\chi_{\max }\right.$ in $\left.\mathrm{cm}^{-1}\right): 1660$ (NHCO), $1706(\mathrm{C}=0), 3420(\mathrm{OH}) .{ }^{1} \mathrm{H}$

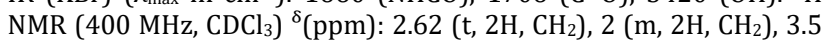
(t, 2HN-CH $\left.\mathrm{CH}_{2}\right), 6.9-8.4(\mathrm{~m}, 16 \mathrm{H}, \mathrm{Ar}-\mathrm{H}), 3.58\left(\mathrm{~s}, 2 \mathrm{H}, \mathrm{CH}_{2}\right), 4.02(\mathrm{~s}, 1 \mathrm{H}$, OH). LCMS: $\mathrm{m} / \mathrm{z}=435[\mathrm{M}]^{+}$. Analysis: Calcd for $\mathrm{C}_{29} \mathrm{H}_{25} \mathrm{NO}_{3}$ (435): $\mathrm{C}$, 79.98; H, 5.79; N, 3.22. Found: C, 7 9.95; H, 5.75; N, 3.20.

\section{3-hydroxy-3-(2-oxo-2-(thiophen-2-yl)ethyl)-1-(3-phenylpropyl)} indolin-2-one (3b)

IR $(\mathrm{KBr})\left(\lambda_{\max }\right.$ in $\left.\mathrm{cm}^{-1}\right): 1630$ (NHCO), $1710(\mathrm{C}=0), 3523(\mathrm{OH}) \cdot{ }^{1} \mathrm{H}$ NMR (400 MHz, $\left.\mathrm{CDCl}_{3}\right)^{8}(\mathrm{ppm}): 2.62(\mathrm{t}, 2 \mathrm{H}, \mathrm{CH} 2), 1.9(\mathrm{~m}, 2 \mathrm{H}, \mathrm{CH} 2)$, $3.5\left(\mathrm{t}, 2 \mathrm{H}, \mathrm{N}-\mathrm{CH}_{2}\right), 6.9-7.9(\mathrm{~m}, 12 \mathrm{H}, \mathrm{Ar}-\mathrm{H}), 4.20(\mathrm{~s}, 1 \mathrm{H}, \mathrm{OH}), 3.7(\mathrm{~s}, 2 \mathrm{H}$, $\mathrm{CH}_{2}$ ). LCMS: $\mathrm{m} / \mathrm{z}=391[\mathrm{M}]^{+}$. Analysis: Calcd for $\mathrm{C}_{23} \mathrm{H}_{21} \mathrm{NO}_{3} \mathrm{~S}$ (391): C, 70.56; H, 5.4; N, 3.58. Found: C, 70.60; H, 5.5; N,3.6.

3-hydroxy-3-(2-oxo-2-(pyridin-3-yl)ethyl)-1-(3-phenylpropyl) indolin-2-one (3c)

IR (KBr) $\left(\chi_{\max }\right.$ in $\left.\mathrm{cm}^{-1}\right): 1670$ (NHCO), $1740(\mathrm{C}=0), 3523(\mathrm{OH}) \cdot{ }^{1} \mathrm{H}$ NMR (400 MHz, $\left.\mathrm{CDCl}_{3}\right)^{\delta}(\mathrm{ppm}): 2.62(\mathrm{t}, 2 \mathrm{H}, \mathrm{CH} 2), 1.9(\mathrm{~m}, 2 \mathrm{H}, \mathrm{CH} 2)$, $3.8\left(\mathrm{t}, 2 \mathrm{H}, \mathrm{N}-\mathrm{CH}_{2}\right)$ 6.9-8.4 (m, $\left.13 \mathrm{H} \mathrm{Ar}-\mathrm{H}\right), 3.3\left(\mathrm{~s}, 2 \mathrm{H}, \mathrm{CH}_{2}\right), 4.08(\mathrm{~s}, 1 \mathrm{H}$, OH). LCMS: $\mathrm{m} / \mathrm{z}=386[\mathrm{M}]^{+}$. Analysis: Calcd for $\mathrm{C}_{24} \mathrm{H}_{22} \mathrm{~N}_{2} \mathrm{O}_{3}$ (386): C, 74.59; H, 5.74; N, 7.25. Found: C, 74.60; H, 5.72; N, 7.20. 3-(2-(4-fluorophenyl)-2-oxoethyl)-3-hydroxy-1-(3phenylpropyl)indolin-2-one (3d)

IR (KBr) $\left(\lambda_{\max }\right.$ in $\left.\mathrm{cm}^{-1}\right): 1640(\mathrm{NHCO}), 1720(\mathrm{C}=0), 3523(\mathrm{OH}) .{ }^{1} \mathrm{H}$ NMR (400 MHz, $\left.\mathrm{CDCl}_{3}\right){ }^{8}(\mathrm{ppm}): 2.62(\mathrm{t}, 2 \mathrm{H}, \mathrm{CH} 2), 1.9(\mathrm{~m}, 2 \mathrm{H}, \mathrm{CH} 2)$, $3.8\left(\mathrm{t}, 2 \mathrm{H}, \mathrm{N}-\mathrm{CH}_{2}\right), 6.91-8.15(\mathrm{~m}, 13 \mathrm{H}, \mathrm{Ar}-\mathrm{H}), 3.50\left(\mathrm{~s}, 2 \mathrm{H}, \mathrm{CH}_{2}\right), 4.18(\mathrm{~s}$, $1 \mathrm{H}, \mathrm{OH})$. LCMS: $\mathrm{m} / \mathrm{z}=403[\mathrm{M}]^{+}$. Analysis: Calcd for $\mathrm{C}_{25} \mathrm{H}_{22} \mathrm{FNO}_{3}$ (403): C, 74.43; H, 5.50; N, 3.47. Found: C, 74.5; H, 5.5, N, 3.45

3-hydroxy-3-(2-(4-methoxyphenyl)-2-oxoethyl)-1-(3phenylpropyl)indolin-2-one (3e)

IR (KBr) $\left(\lambda_{\max }\right.$ in cm-1): 1630 (NHCO), 1710 (C=0), $3500(\mathrm{OH}) .{ }^{1} \mathrm{H}$ NMR $\left(400 \mathrm{MHz}, \mathrm{CDCl}_{3}\right)^{\delta}(\mathrm{ppm}): 2.62\left(\mathrm{t}, 2 \mathrm{H}, \mathrm{CH}_{2}\right), 1.9\left(\mathrm{~m}, 2 \mathrm{H}, \mathrm{CH}_{2}\right), 3.8(\mathrm{t}, 2 \mathrm{H}$, $\left.\mathrm{N}-\mathrm{CH}_{2}\right)$ 6.9-7.8 (m, 13H, Ar-H), $3.3\left(\mathrm{~s}, 2 \mathrm{H}, \mathrm{CH}_{2}\right), 4.24(\mathrm{~s}, 1 \mathrm{H}, \mathrm{OH}), 3.83$ (s, $3 \mathrm{H}, \mathrm{OCH}_{3}$ ). LCMS: $\mathrm{m} / \mathrm{z}=415$ [M] ${ }^{+}$. Analysis: Calcd for $\mathrm{C}_{26} \mathrm{H}_{25} \mathrm{NO}_{4}$ (415): C, 75.16; H, 6.06; N, 3.37. Found: C, 75.16; H, 6.05, N, 3.4.

\section{4-(2-(3-hydroxy-2-oxo-1-(3-phenylpropyl)indolin-3-yl)acetyl) benzonitrile ( $3 f$ )}

IR (KBr) $\left(\lambda_{\max }\right.$ in $\left.\mathrm{cm}^{-1}\right): 1645$ (NHCO), $1720(\mathrm{C}=0), 3523(\mathrm{OH}) .{ }^{1} \mathrm{H}$ NMR $\left(400 \mathrm{MHz}, \mathrm{CDCl}_{3}\right)^{\delta}(\mathrm{ppm}): 2.62\left(\mathrm{t}, 2 \mathrm{H}, \mathrm{CH}_{2}\right), 1.9(\mathrm{~m}, 2 \mathrm{H}, \mathrm{CH} 2), 3.8(\mathrm{t}, 2 \mathrm{H}$, $\left.\mathrm{N}-\mathrm{CH}_{2}\right)$ ) 6.51-8.11 (m, $\left.13 \mathrm{H}, \mathrm{Ar}-\mathrm{H}\right), 3.78\left(\mathrm{~s}, 2 \mathrm{H}, \mathrm{CH}_{2}\right), 4.20(\mathrm{~s}, 1 \mathrm{H}, \mathrm{OH})$. LCMS: $\mathrm{m} / \mathrm{z}=411[\mathrm{M}]^{+}$. Analysis: Calcd for $\mathrm{C}_{26} \mathrm{H}_{22} \mathrm{~N}_{2} \mathrm{O}_{3}$ (411): C, 76.08; H, 5.40; N, 6.82; O, 11.69. Found: C, 76.07; H, 5.32; N, 6.84; 0, 11.67 .

5-fluoro-3-hydroxy-3-(2-(naphthalen-2-yl)-2-oxoethyl)-1-(3phenylpropyl)indolin-2-one (3g)

IR (KBr) ( $\chi_{\max }$ in $\left.\mathrm{cm}^{-1}\right): 1660$ (NHCO), 1706(C=0), $3500(\mathrm{OH}) .{ }^{1} \mathrm{H}$ NMR (400 MHz, $\left.\mathrm{CDCl}_{3}\right)^{8}(\mathrm{ppm}): 2.62\left(\mathrm{t}, 2 \mathrm{H}, \mathrm{CH}_{2}\right), 2\left(\mathrm{~m}, 2 \mathrm{H}, \mathrm{CH}_{2}\right), 3.5$ (t, $\left.2 \mathrm{H}, \mathrm{N}-\mathrm{CH}_{2}\right), 6.9-8.4(\mathrm{~m}, 15 \mathrm{H}, \mathrm{Ar}-\mathrm{H}), 3.25\left(\mathrm{~s}, 2 \mathrm{H}, \mathrm{CH}_{2}\right), 4.24(\mathrm{~s}, 1 \mathrm{H}$, $\mathrm{OH}$. LCMS: $\mathrm{m} / \mathrm{z}=453$ [M] $]^{+}$. Analysis: Calcd for $\mathrm{C}_{29} \mathrm{H}_{24} \mathrm{FNO}_{3}$ (453): $\mathrm{C}$, 76.80; H, 5.33; N, 3.09. Found: C, 76.81; H, 5.34; N, 3.08.

5-fluoro-3-hydroxy-3-(2-oxo-2-(thiophen-2-yl)ethyl)-1-(3phenylpropyl)indolin-2-one (3h)

IR (KBr) ( $\chi_{\max }$ in $\left.\mathrm{cm}^{-1}\right): 1630(\mathrm{NHCO}), 1710(\mathrm{C}=0), 3523(\mathrm{OH}) .{ }^{1} \mathrm{H}$ NMR (400 MHz, $\left.\mathrm{CDCl}_{3}\right){ }^{8}(\mathrm{ppm}): 2.62\left(\mathrm{t}, 2 \mathrm{H}, \mathrm{CH}_{2}\right), 1.9\left(\mathrm{~m}, 2 \mathrm{H}, \mathrm{CH}_{2}\right)$, $3.5\left(\mathrm{t}, 2 \mathrm{H}, \mathrm{N}-\mathrm{CH}_{2}\right), 6.9-7.9(\mathrm{~m}, 11 \mathrm{H}, \mathrm{Ar}-\mathrm{H}), 3.75\left(\mathrm{~s}, 2 \mathrm{H}, \mathrm{CH}_{2}\right), 4.20(\mathrm{~s}$, 1H, OH). LCMS: $\mathrm{m} / \mathrm{z}=409[\mathrm{M}]^{+}$. Analysis: Calcd for $\mathrm{C}_{23} \mathrm{H}_{20} \mathrm{FNO}_{3} \mathrm{~S}$ (409): C, 67.46; H, 4.92; N, 3.42. Found: C, 67.43; H, 4.95; N, 3.44.

5-flouro-3-hydroxy-3-(2-oxo-2-(pyridin-3-yl)ethyl)-1-(3phenylpropyl)indolin-2-one (3i)

IR (KBr) $\left(\chi_{\max }\right.$ in $\left.\mathrm{cm}^{-1}\right): 1670(\mathrm{NHCO}), 1740(\mathrm{C}=0), 3523(\mathrm{OH}) .{ }^{1} \mathrm{H}$ NMR $\left(400 \mathrm{MHz}, \mathrm{CDCl}_{3}\right){ }^{8}(\mathrm{ppm}): 2.62\left(\mathrm{t}, 2 \mathrm{H}, \mathrm{CH}_{2}\right), 1.9\left(\mathrm{~m}, 2 \mathrm{H}, \mathrm{CH}_{2}\right)$, $3.8\left(\mathrm{t}, 2 \mathrm{H}, \mathrm{N}-\mathrm{CH}_{2}\right), 6.9-8.4(\mathrm{~m}, 12 \mathrm{H}, \mathrm{Ar}-\mathrm{H}), 3.5\left(\mathrm{~s}, 2 \mathrm{H}, \mathrm{CH}_{2}\right), 4.15(\mathrm{~s}, 1 \mathrm{H}$, OH). LCMS: $\mathrm{m} / \mathrm{z}=404[\mathrm{M}]^{+}$. Analysis: Calcd for $\mathrm{C}_{24} \mathrm{H}_{21} \mathrm{FN}_{2} \mathrm{O}_{3}$ (404): C, 71.27; H, 5.23; N, 6.93. Found: C, 71.29; H, 5.24; N, 6.95.

5-flouro-3-(2-(4-fluorophenyl)-2-oxoethyl)-3-hydroxy-1-(3phenylpropyl)indolin-2-one $(3 \mathrm{j})$

IR (KBr) ( $\lambda_{\max }$ in cm-1): $1640(\mathrm{NHCO}), 1685(\mathrm{C}=0), 3523(\mathrm{OH}) .{ }^{1} \mathrm{H}$ NMR $\left(400 \mathrm{MHz}, \mathrm{CDCl}_{3}\right){ }^{8}(\mathrm{ppm}): 2.62\left(\mathrm{t}, 2 \mathrm{H}, \mathrm{CH}_{2}\right), 1.9\left(\mathrm{~m}, 2 \mathrm{H}, \mathrm{CH}_{2}\right)$, $3.8\left(\mathrm{t}, 2 \mathrm{H}, \mathrm{N}-\mathrm{CH}_{2}\right), 6.91-8.15(\mathrm{~m}, 12 \mathrm{H}, \mathrm{Ar}-\mathrm{H}), 3.78\left(\mathrm{~s}, 2 \mathrm{H}, \mathrm{CH}_{2}\right), 4.20(\mathrm{~s}$, $1 \mathrm{H}, \mathrm{OH})$. LCMS: $\mathrm{m} / \mathrm{z}=421[\mathrm{M}]^{+}$. Analysis: Calcd for $\mathrm{C}_{25} \mathrm{H}_{21} \mathrm{~F}_{2} \mathrm{NO}_{3}$ (421): C, 71.25; H, 5.02; N, 3.3. Found: C, 71.27; H, 5.04; N,3.35.

5-flouro-3-hydroxy-3-(2-(4-methoxyphenyl)-2-oxoethyl)-1-(3phenylpropyl)indolin-2-one (3k)

IR (KBr) ( $\lambda_{\max }$ in $\left.\mathrm{cm}^{-1}\right): 1630(\mathrm{NHCO}), 1710(\mathrm{C}=0), 3500(\mathrm{OH}) .{ }^{1} \mathrm{H}$ NMR (400 MHz, $\left.\mathrm{CDCl}_{3}\right){ }^{\delta}(\mathrm{ppm}): 2.62\left(\mathrm{t}, 2 \mathrm{H}, \mathrm{CH}_{2}\right), 1.9\left(\mathrm{~m}, 2 \mathrm{H}, \mathrm{CH}_{2}\right)$, $3.8\left(\mathrm{t}, 2 \mathrm{H}, \mathrm{N}-\mathrm{CH}_{2}\right), 6.9-7.8(\mathrm{~m}, 12 \mathrm{H}, \mathrm{Ar}-\mathrm{H}), 3.03\left(\mathrm{~s}, 2 \mathrm{H}, \mathrm{CH}_{2}\right), 4.65(\mathrm{~s}$, $1 \mathrm{H}, \mathrm{OH}), 3.83\left(\mathrm{~s}, 3 \mathrm{H}, \mathrm{OCH}_{3}\right)$. LCMS: $\mathrm{m} / \mathrm{z}=435[\mathrm{M}]^{+}$. Analysis: Calcd for $\mathrm{C}_{26} \mathrm{H}_{24} \mathrm{FNO}_{4}$ (435): C, 72.04; H, 5.58; N, 3.23. Found: C, 72.06; H, 5.

5-flouro-4-(2-(3-hydroxy-2-oxo-1-(3-phenylpropyl)indolin-3yl)acetyl)benzonitrile (31)

IR (KBr) $\left(\lambda_{\max }\right.$ in $\left.\mathrm{cm}^{-1}\right): 1670(\mathrm{NHCO}) ; 1740(\mathrm{C}=0) ; 3523(\mathrm{OH}) .{ }^{1} \mathrm{H}$ NMR (400 MHz, $\left.\mathrm{CDCl}_{3}\right): 2.62\left(\mathrm{t}, 2 \mathrm{H}, \mathrm{CH}_{2}\right), 1.9\left(\mathrm{~m}, 2 \mathrm{H}, \mathrm{CH}_{2}\right), 3.8(\mathrm{t}, 2 \mathrm{H}$, $\left.\mathrm{N}-\mathrm{CH}_{2}\right), 6.9-8.4(\mathrm{~m}, 12 \mathrm{H}, \mathrm{Ar}-\mathrm{H}), 3.5\left(\mathrm{~s}, 2 \mathrm{H}, \mathrm{CH}_{2}\right), 4.15(\mathrm{~s}, 1 \mathrm{H}, \mathrm{OH})$. LCMS: $\mathrm{m} / \mathrm{z}=428[\mathrm{M}]^{+}$. Analysis: Calcd for $\mathrm{C}_{26} \mathrm{H}_{21} \mathrm{FN}_{2} \mathrm{O}_{3}$ (428): C, 72.88; H, 4.94; N,6.54. Found: C, 71.89; H, 4.96; N, 6.55. 
General procedure for the synthesis of 3-(2-oxoethylidene)-1(3-phenylpropyl)indolin-2-one derivatives 4(a-l)

To a stirred solution of 3(a-l) $(0.91 \mathrm{mmol})$ in ethanol $(15 \mathrm{ml})$ was added concentrated $\mathrm{HCl}(5 \mathrm{ml})$ and the reaction mixture was refluxed for $6 \mathrm{~h}$. The progress of the reaction was monitored on TLC using several solvent systems of different polarity. Reaction mixture was filtered, dried and purified by recrystallization from ethanol to obtain the desired product as bright red needles.

\section{3-(2-(naphthalen-2-yl)-2-oxoethylidene)-1-(3-} phenylpropyl)indolin-2-one (4a)

IR (KBr) $\left(\lambda_{\max }\right.$ in $\left.\mathrm{cm}^{-1}\right): 1660(\mathrm{NHC}=0), 1706(\mathrm{C}=0), 3059$ (Ar C-C stretch). ${ }^{1} \mathrm{H}$ NMR $\left(400 \mathrm{MHz}, \mathrm{CDCl}_{3}\right)^{\delta}(\mathrm{ppm}): 2.07-2.79\left(\mathrm{~m}, 2 \mathrm{H}, \mathrm{CH}_{2}\right)$, $2.78\left(\mathrm{t}, 2 \mathrm{H}, \mathrm{CH}_{2}\right), 3.85\left(\mathrm{t}, 2 \mathrm{H}, \mathrm{N}-\mathrm{CH}_{2}\right), 6.74(\mathrm{~s}, 1 \mathrm{H}, \mathrm{CH}), 7.04-8.6(\mathrm{~m}$, $16 \mathrm{H}, \mathrm{Ar}-\mathrm{H})$. LCMS: $\mathrm{m} / \mathrm{z}=417[\mathrm{M}]^{+}$. Analysis: Calcd for $\mathrm{C}_{29} \mathrm{H}_{23} \mathrm{NO}_{2}$ (417): C, 83.43; H, 5.55; N, 3.35. Found: C, 83.93; H, 5.58; N, 3.39.

\section{3-(2-oxo-2-(thiophen-2-yl)ethylidene)-1-(3-phenylpropyl)} indolin-2-one (4b)

IR $(\mathrm{KBr})\left(\lambda_{\max }\right.$ in $\left.\mathrm{cm}^{-1}\right): 1649(\mathrm{NHC}=0), 1711(\mathrm{C}=0), 3080(\mathrm{Ar} \mathrm{C}-\mathrm{H}$ stretch). 650 (C-S). ${ }^{1} \mathrm{H}$ NMR (400 MHz, $\left.\mathrm{CDCl}_{3}\right){ }^{8}(\mathrm{ppm}): 2.06(\mathrm{~m}, 2 \mathrm{H}$, $\left.\mathrm{CH}_{2}\right), 2.75\left(\mathrm{t}, 2 \mathrm{H}, \mathrm{CH}_{2}\right), 3.82\left(\mathrm{t}, 2 \mathrm{H}, \mathrm{N}-\mathrm{CH}_{2}\right), 6.71(\mathrm{~s}, 1 \mathrm{H} . \mathrm{CH}), 7.07-8.5$ (m, $12 \mathrm{H}, \mathrm{Ar}-\mathrm{H})$. LCMS: $\mathrm{m} / \mathrm{z}=373[\mathrm{M}]^{+}$. Analysis: Calcd for $\mathrm{C}_{29} \mathrm{H}_{19}$ $\mathrm{NO}_{2} \mathrm{~S}$ (373): C, 73.97; H, 5.13; N, 3.75. Found: C, 73.97; H, 5.14, N, 3.79 .

\section{3-(2-oxo-2-(pyridin-3-yl)ethylidene)-1-(3-phenylpropyl)} indolin-2-one (4c)

IR (KBr) $\left(\lambda_{\max }\right.$ in $\left.\mathrm{cm}^{-1}\right): 1660(\mathrm{R}-\mathrm{C}=0), 1706(\mathrm{C}=0), 3018(\mathrm{Ar} \mathrm{C}-\mathrm{H}$ stretch), $1608(\mathrm{C}=\mathrm{N})$.

${ }^{1} \mathrm{H}$ NMR $\left(400 \mathrm{MHz}, \mathrm{CDCl}_{3}\right){ }^{\delta}(\mathrm{ppm}): 2.05\left(\mathrm{~m}, 2 \mathrm{H}, \mathrm{CH}_{2}\right), 2.75(\mathrm{t}, 2 \mathrm{H}$, $\left.\mathrm{CH}_{2}\right), 3.81\left(\mathrm{t}, 2 \mathrm{H}, \mathrm{N}-\mathrm{CH}_{2}\right), 6.72\left(\mathrm{~s}, 1 \mathrm{H}, \mathrm{CH}_{2}\right)$ 7.03-8.85 (m, 13H, Ar-H). LCMS: $\mathrm{m} / \mathrm{z}=368$ [M] ${ }^{+}$. Calcd for $\mathrm{C}_{24} \mathrm{H}_{20} \mathrm{~N}_{2} \mathrm{O}_{2}$ (368): C, 78.24; $\mathrm{H}, 5.47$; N, 6.70. Found: C, 78.28; H, 5.57; N, 6.72.

\section{3-(2-(4-fluorophenyl)-2-oxoethylidene)-1-(3-} phenylpropyl)indolin-2-one (4d)

IR $(\mathrm{KBr})\left(\chi_{\max }\right.$ in $\left.\mathrm{cm}^{-1}\right): 1660(\mathrm{NHC}=0), 1706(\mathrm{C}=0), 3054(\mathrm{Ar} \mathrm{C}-\mathrm{H}$ stretch), 844 (C-F stretch). ${ }^{1} \mathrm{H}$ NMR $\left(400 \mathrm{MHz}, \mathrm{CDCl}_{3}\right)^{\delta}(\mathrm{ppm}): 2.08$ $\left(\mathrm{m}, 2 \mathrm{H}, \mathrm{CH}_{2}\right), 2.75\left(\mathrm{t}, 2 \mathrm{H}, \mathrm{CH}_{2}\right), 3.82\left(\mathrm{t}, 2 \mathrm{H}, \mathrm{N}-\mathrm{CH}_{2}\right), 7.1(\mathrm{~s}, 1 \mathrm{H}, \mathrm{CH})$, 7.3-7.6 (m, 13H, Ar-H). LCMS: $\mathrm{m} / \mathrm{z}=385$ [M] ${ }^{+}$. Calcd for $\mathrm{C}_{25} \mathrm{H}_{20} \mathrm{FNO}_{2}$ (385): C, 77.90; H, 5.23; N, 3.63. Found: C, 77.92; H, 5.25; N, 3.69.

\section{3-(2-(4-methoxyphenyl)-2-oxoethylidene)-1-(3-} phenylpropyl)indolin-2-one (4e)

IR (KBr) $\left(\lambda_{\max }\right.$ in $\left.\mathrm{cm}^{-1}\right): 1655(\mathrm{NHC}=0), 1706(\mathrm{C}=0), 3059(\mathrm{Ar} \mathrm{C}-\mathrm{H}$ stretch), 1598 (C=C stretch). ${ }^{1} \mathrm{H}$ NMR $\left(400 \mathrm{MHz}^{\left.-\mathrm{CDCl}_{3}\right)}{ }^{8}(\mathrm{ppm}): 2.09\right.$ $\left(\mathrm{m}, 2 \mathrm{H}, \mathrm{CH}_{2}\right), 2.75\left(\mathrm{t}, 2 \mathrm{H}, \mathrm{CH}_{2}\right), 3.82\left(\mathrm{t}, 2 \mathrm{H}, \mathrm{N}-\mathrm{CH}_{2}\right), 3.92\left(\mathrm{~s}, 3 \mathrm{H} . \mathrm{OCH}_{3}\right)$, $6.72(\mathrm{~s}, 1 \mathrm{H}, \mathrm{CH}), 7.27-8.1(\mathrm{~m}, 13 \mathrm{H}, \mathrm{Ar}-\mathrm{H})$. LCMS: $\mathrm{m} / \mathrm{z}=397[\mathrm{M}]^{+}$. Analysis: Calcd for $\mathrm{C}_{26} \mathrm{H}_{23} \mathrm{NO}_{3}$ (397): C, 78.57; H, 5.83; N,3.52. Found: C, 78.59; H, 5.85, N, 3.54 .

\section{4-(2-(2-oxo-1-(3-phenylpropyl)indolin-3-ylidene)acetyl)} benzonitrile (4f)

IR $(\mathrm{KBr})\left(\lambda_{\max }\right.$ in $\left.\mathrm{cm}^{-1}\right): 1650(\mathrm{NHC}=0), 1710(\mathrm{C}=0), 3090(\mathrm{Ar} \mathrm{C}-\mathrm{H}$ stretch). ${ }^{1} \mathrm{H}$ NMR $\left(400 \mathrm{MHz}, \mathrm{CDCl}_{3}\right){ }^{8}(\mathrm{ppm}): 2.06\left(\mathrm{~m}, 2 \mathrm{H}, \mathrm{CH}_{2}\right), 2.74$ $\left(\mathrm{t}, 2 \mathrm{H}, \mathrm{CH}_{2}\right), 3.81\left(\mathrm{t}, 2 \mathrm{H}, \mathrm{N}-\mathrm{CH}_{2}\right), 7.75(\mathrm{~s}, 1 \mathrm{H}, \mathrm{CH}), 6.9-8.1(\mathrm{~m}, 13 \mathrm{H}, \mathrm{Ar}-$ H). LCMS: $\mathrm{m} / \mathrm{z}=392[\mathrm{M}]^{+}$. Analysis: Calcd for $\mathrm{C}_{26} \mathrm{H}_{20} \mathrm{~N}_{2} \mathrm{O}_{2}$ (392): $\mathrm{C}$, 79.57; H, 5.14; N, 7.14. Found: C, 79.59; H, 5.15; N, 7.16.

5-flouro-3-(2-(naphthalen-2-yl)-2-oxoethylidene)-1-(3phenylpropyl)indolin-2-one (4g)

IR (KBr) ( $\chi_{\max }$ in $\left.\mathrm{cm}^{-1}\right): 1660(\mathrm{NHC}=0), 1711(\mathrm{C}=0), 3059$ (Ar C-H stretch), $1678\left(\mathrm{C}=\mathrm{C}\right.$ stretch). ${ }^{1} \mathrm{H}$ NMR $\left(400 \mathrm{MHz}, \mathrm{CDCl}_{3}\right){ }^{8}(\mathrm{ppm}): 2.09$ $\left(\mathrm{m}, 2 \mathrm{H}, \mathrm{CH}_{2}\right), 2.74\left(\mathrm{t}, 2 \mathrm{H}, \mathrm{CH}_{2}\right), 3.8\left(\mathrm{t}, 2 \mathrm{H}, \mathrm{N}-\mathrm{CH}_{2}\right), 6.6(\mathrm{~s}, 1 \mathrm{H}, \mathrm{CH})$ 7.27-8.5 (m, 15H, Ar-H), LCMS: $\mathrm{m} / \mathrm{z}=435[\mathrm{M}]^{+}$. Analysis: Calcd for
$\mathrm{C}_{29} \mathrm{H}_{22} \mathrm{FNO}_{2}$ (435): C, 79.98; H, 5.09; N, 3.22. Found: C, 79.99; H, 5.10; $\mathrm{N}, 3.25$.

5-fluoro-3-(2-oxo-2-(thiophen-2-yl)ethylidene)-1-(3phenylpropyl)indolin-2-one (4h)

IR (KBr) $\left(\chi_{\max }\right.$ in $\left.\mathrm{cm}^{-1}\right): 1649(\mathrm{NHC}=0), 1706(\mathrm{C}=0), 3090(\mathrm{Ar} \mathrm{C}-\mathrm{H}$ stretch). ${ }^{1} \mathrm{H}$ NMR $\left(400 \mathrm{MHz}, \mathrm{CDCl}_{3}\right){ }^{8}(\mathrm{ppm}): 2.06\left(\mathrm{~m}, 2 \mathrm{H}, \mathrm{CH}_{2}\right), 2.74$ $\left(\mathrm{t}, 2 \mathrm{H}, \mathrm{CH}_{2}\right), 3.81\left(\mathrm{t}, 2 \mathrm{H}, \mathrm{N}-\mathrm{CH}_{2}\right), 6.63(\mathrm{~s}, 1 \mathrm{H}, \mathrm{CH}), 7.07-8.4(\mathrm{~m}, 11 \mathrm{H}$, Ar-H). LCMS: $\mathrm{m} / \mathrm{z}=391[\mathrm{M}]^{+}$. Analysis: Calcd for $\mathrm{C}_{23} \mathrm{H}_{18} \mathrm{FNO}_{2} \mathrm{~S}$ (391): C, 70.58; H, 4.63; N, 3.58. Found: C, 70.59; H, 4.65; N, 3.60 .

5-flouro-3-(2-oxo-2-(pyridin-3-yl)ethylidene)-1-(3phenylpropyl) indolin-2-one (4i)

IR (KBr) $\left(\chi_{\max }\right.$ in $\left.\mathrm{cm}^{-1}\right): 1660(\mathrm{R}-\mathrm{C}=0), 1706(\mathrm{C}=0), 3018(\mathrm{Ar} \mathrm{C}-\mathrm{H}$ stretch), $1608(\mathrm{C}=\mathrm{N}) .{ }^{1} \mathrm{H}$ NMR $\left(400 \mathrm{MHz}, \mathrm{CDCl}_{3}\right){ }^{8}(\mathrm{ppm}): 2.02(\mathrm{~m}, 2 \mathrm{H}$, $\left.\mathrm{CH}_{2}\right), 2.74\left(\mathrm{t}, 2 \mathrm{H}, \mathrm{CH}_{2}\right), 3.79\left(\mathrm{t}, 2 \mathrm{H}, \mathrm{N}-\mathrm{CH}_{2}\right), 6.7(\mathrm{~s}, 1 \mathrm{H} . \mathrm{CH}), 7.03-8.85$ $(\mathrm{m}, 12 \mathrm{H}, \mathrm{Ar}-\mathrm{H})$. LCMS: $\mathrm{m} / \mathrm{z}=386[\mathrm{M}]^{+}$. Analysis: Calcd for $\mathrm{C}_{24} \mathrm{H}_{19} \mathrm{FN}_{2} \mathrm{O}_{2}$ (386): C, 74.60; $\mathrm{H}, 4.96 ; \mathrm{N}, 7.25$. Found: $\mathrm{C}, 74.64 ; \mathrm{H}$, 4.99; N,7.28.

5-fluoro-3-(2-(4-fluorophenyl)-2-oxoethylidene)-1-(3phenylpropyl)indolin-2-one $(4 \mathrm{j})$

IR (KBr) ( $\chi_{\max }$ in $\left.\mathrm{cm}^{-1}\right): 1665(\mathrm{NHC}=0), 1713(\mathrm{C}=0), 3070(\mathrm{Ar} \mathrm{C}-\mathrm{H}$ stretch). 699 (C-F). ${ }^{1} \mathrm{H}$ NMR $\left(400 \mathrm{MHz}, \mathrm{CDCl}_{3}\right){ }^{\delta}(\mathrm{ppm}): 2.02(\mathrm{~m}, 2 \mathrm{H}$, $\left.\mathrm{CH}_{2}\right), 2.6\left(\mathrm{t}, 2 \mathrm{H}, \mathrm{CH}_{2}\right), 3.79\left(\mathrm{t}, 2 \mathrm{H}, \mathrm{N}-\mathrm{CH}_{2}\right), 7(\mathrm{~s}, 1 \mathrm{H}, \mathrm{CH}), 7.2-7.8(\mathrm{~m}$, $12 \mathrm{H}, \mathrm{Ar}-\mathrm{H}$ ). LCMS: $\mathrm{m} / \mathrm{z}=403[\mathrm{M}]^{+}$. Analysis: Calcd for $\mathrm{C}_{25} \mathrm{H}_{19} \mathrm{~F}_{2} \mathrm{NO}_{2}$ (403): C, 74.34; H, 4.75; N, 3.47. Found: C, 74.38; H, 4.71; N, 3.48.

5-flouro-3-(2-(4-methoxyphenyl)-2-oxoethylidene)-1-(3phenylpropyl)indolin-2-one (4k)

IR $(\mathrm{KBr})\left(\chi_{\max }\right.$ in $\left.\mathrm{cm}^{-1}\right): 1655(\mathrm{NHC}=0), 1700(\mathrm{C}=0), 3020(\mathrm{Ar} \mathrm{C}-\mathrm{H}$ stretch), 690 (C-F). ${ }^{1} \mathrm{H}$ NMR (400 MHz, $\left.\mathrm{CDCl}_{3}\right){ }^{\delta}(\mathrm{ppm}): 2.09(\mathrm{~m}, 2 \mathrm{H}$, $\left.\mathrm{CH}_{2}\right), 2.75\left(\mathrm{t}, 2 \mathrm{H}, \mathrm{CH}_{2}\right), 3.7\left(\mathrm{t}, 2 \mathrm{H}, \mathrm{N}-\mathrm{CH}_{2}\right), 6.7(\mathrm{~s}, 1 \mathrm{H}, \mathrm{CH}), 7.27-8.1(\mathrm{~m}$, $12 \mathrm{H}, \mathrm{Ar}-\mathrm{H}), 3.83\left(\mathrm{~s}, 3 \mathrm{H}, \mathrm{OCH}_{3}\right)$. LCMS: $\mathrm{m} / \mathrm{z}=415[\mathrm{M}]^{+}$. Analysis: Calcd for $\mathrm{C}_{26} \mathrm{H}_{22} \mathrm{FNO}_{3}$ (415): C, 75.17; $\mathrm{H}, 5.34 ; \mathrm{N}, 3.37$. Found: $\mathrm{C}, 75.17 ; \mathrm{H}$, $5.34 ; \mathrm{N}, 3.37$.

5-flouro-4-(2-(2-oxo-1-(3-phenylpropyl)indolin-3ylidene)acetyl)benzonitrile (4I)

IR $(\mathrm{KBr})\left(\chi_{\max }\right.$ in $\left.\mathrm{cm}^{-1}\right): 1650(\mathrm{NHC}=0), 1710(\mathrm{C}=0), 3090(\mathrm{Ar} \mathrm{C}-\mathrm{H}$ stretch). ${ }^{1} \mathrm{H}$ NMR $\left(400 \mathrm{MHz}, \mathrm{CDCl}_{3}\right){ }^{8}(\mathrm{ppm}): 2.06\left(\mathrm{~m}, 2 \mathrm{H}, \mathrm{CH}_{2}\right), 2.74$ $\left(\mathrm{t}, 2 \mathrm{H}, \mathrm{CH}_{2}\right), 3.81\left(\mathrm{t}, 2 \mathrm{H}, \mathrm{N}-\mathrm{CH}_{2}\right), 6.72(\mathrm{~s}, 1 \mathrm{H}, \mathrm{CH}), 6.9-8.1(\mathrm{~m}, 12 \mathrm{H}, \mathrm{Ar}-$ H). LCMS: $\mathrm{m} / \mathrm{z}=410[\mathrm{M}]^{+}$. Analysis: Calcd for $\mathrm{C}_{26} \mathrm{H}_{19} \mathrm{FN}_{2} \mathrm{O}_{2}$ (410): $\mathrm{C}$, 76.08; H, 4.67; N, 6.83. Found: C, 76.08; H, 4.67; N, 6.83.

\section{Biological activities}

\section{Antimicrobial activity}

The antibacterial activities of compounds $4(\mathrm{a}-\mathrm{l})$, were carried out using the Cup plate diffusion method [25]. This method depends on the diffusion of the antibiotic from a cavity through the solidified agar layer in a petri dish to an extent such that the growth of the added microorganism is prevented in a circular zone around the cavity containing a solution of the antibiotic. For antibacterial activity, antibacterial species used are two Gram negative species, Escherichia coli (ATCC 9637), Salmonella typhi (ATCC 6539) and two Gram-positive species, Bacillus subtilis (ATCC 6633), Staphylococcus aureus (ATCC 29737). Two fungal strains Aspergillus niger (ATCC 16509), Aspergillus fumigates (ATCC16406) were used for antifungal activity. Solution of each compound at a concentration of $1000 \mu \mathrm{g} / \mathrm{ml}$ in DMSO was prepared and the inhibition zone diameter in millimeter was used as the criterion for measuring the microbial activity after $24 \mathrm{~h}$ for bacteria and $72 \mathrm{~h}$ for fungi. Ciprofloxacin is used as bacterial standards and Amphotericin B is used as fungal standards for references to evaluate the efficacy of the tested compounds under the same conditions. DMSO used as control and solvent to prepare compound solutions. Measurements of results are shown in table 2 
Table 1: Physical Properties of 3-(2-oxoethylidene)-1-(3-phenylpropyl)indolin-2-one derivatives 4(a-1)

\begin{tabular}{|c|c|c|c|c|c|}
\hline Comp. No & $\begin{array}{ll}\mathbf{R} & \mathbf{R}_{1} \\
\end{array}$ & M. For. & M. Wt. & $\%$ Yield & M. P. ${ }^{\circ} \mathrm{C}$ \\
\hline $4 a$ & $\mathrm{H}$ & $\mathrm{C}_{29} \mathrm{H}_{23} \mathrm{NO}_{2}$ & 417 & $67 \%$ & $149-51$ \\
\hline $4 b$ & $\mathrm{H}$ & $\mathrm{C}_{23} \mathrm{H}_{19} \mathrm{NO}_{2} \mathrm{~S}$ & 373 & $60 \%$ & $172-73$ \\
\hline $4 c$ & $\mathrm{H}$ & $\mathrm{C}_{24} \mathrm{H}_{20} \mathrm{~N}_{2} \mathrm{O}_{2}$ & 368 & $63 \%$ & $160-62$ \\
\hline $4 d$ & $\mathrm{H}$ & $\mathrm{C}_{25} \mathrm{H}_{20} \mathrm{FNO}_{2}$, & 385 & $67 \%$ & $173-75$ \\
\hline $4 \mathrm{e}$ & $\mathrm{H}$ & $\mathrm{C}_{26} \mathrm{H}_{23} \mathrm{NO}_{3}$ & 397 & $67 \%$ & $146-47$ \\
\hline $4 \mathrm{f}$ & $\mathrm{H}$ & $\mathrm{C}_{26} \mathrm{H}_{20} \mathrm{~N}_{2} \mathrm{O}_{2}$ & 392 & $58 \%$ & $187-89$ \\
\hline $4 g$ & $\mathrm{~F}$ & $\mathrm{C}_{29} \mathrm{H}_{22} \mathrm{FNO}_{2}$ & 436 & $83 \%$ & 204-05 \\
\hline $4 h$ & $\mathrm{~F}$ & $\mathrm{C}_{23} \mathrm{H}_{18} \mathrm{FNO}_{2} \mathrm{~S}$ & 391 & $62 \%$ & $154-56$ \\
\hline $4 i$ & $\mathrm{~F}$ & $\mathrm{C}_{24} \mathrm{H}_{19} \mathrm{FN}_{2} \mathrm{O}_{2}$ & 386 & $63 \%$ & $210-12$ \\
\hline $4 j$ & $\mathrm{~F}$ & $\mathrm{C}_{25} \mathrm{H}_{19} \mathrm{~F}_{2} \mathrm{NO}_{2}$ & 403 & $56 \%$ & $194-95$ \\
\hline $4 \mathrm{k}$ & $\mathrm{F}$ & $\mathrm{C}_{26} \mathrm{H}_{22} \mathrm{FNO}_{3}$ & 415 & $64 \%$ & $140-42$ \\
\hline 41 & $\mathrm{~F}$ & $\mathrm{C}_{26} \mathrm{H}_{19} \mathrm{FN}_{2} \mathrm{O}_{2}$ & 410 & $61 \%$ & $197-99$ \\
\hline
\end{tabular}

Comp. No.-Compound number. M. for.-Molecular formula, M. wt.-Molecular weight, M. pt.-Melting point.

\section{Antioxidant activity assay}

\section{1, 1-diphenyl-2-picryl hydrazyl (DPPH) radical scavenging activity (RSA)}

The free radical scavenging activity (RSA) of all the compounds at concentrations of $25,50,75$ and $100 \mu \mathrm{g} / \mathrm{ml}$ was carried out in the presence of a freshly prepared solution of stable free radical DPPH $(0.04 \% \mathrm{w} / \mathrm{v})$ following a Hatano's method [26,27]. Ascorbic acid (AA) is used as standards. All the test analyses were performed on three replicates and the results are averaged. The results in percentage are expressed as the ratio of absorption decrease of $\mathrm{DPPH}$ in the presence of test compounds and absorption of DPPH in the absence of test compounds at $\lambda 517 \mathrm{~nm}$ on ELICO SL 171 Mini Spec, spectrophotometer. The percentage scavenging activity of the DPPH free radical was measured using the following equation:

$$
\% \text { of DPPH RSA }=\frac{\text { Absorbance of Control }- \text { Absorbance of Sample }}{\times 10 \quad \text { Absorbance of Control }}
$$

\section{RESULTS AND DISCUSSION}

\section{Chemistry}

The Synthesis of title compounds was on account of the biological activity of indole and was carried out using a general, simple and straight forward pathway. 5-Substituted-1H-indole-2,3-dione was used as basic material for the synthesis of resultant derivatives. The treatment of 5-substituted $1 H$-indole-2,3-dione and (3chloropropyl) benzene in $\mathrm{N}, \mathrm{N}$-Dimethyl formamide with $\mathrm{K}_{2} \mathrm{CO}_{3}$ yield substituted $\mathrm{N}$-phenyl propyl isatins $2(\mathrm{a}-\mathrm{b})$. These on condensation with acetphenone analogues resulted into compounds $3(\mathrm{a}-\mathrm{l})$ which on dehydration yielded compounds 4(a-l) (Scheme 1).
The structure elucidation of the final products was carried out by IR, ${ }^{1} \mathrm{H}-\mathrm{NMR}$ and Mass spectral data. IR peaks of the compound were recognized from $1700-1720 \mathrm{~cm}^{-1}$ for $\mathrm{C}=0$ stretching, $1640-1660 \mathrm{~cm}$ ${ }^{1}$ for NHCO stretching, $3075-2850 \mathrm{~cm}^{-1}$ for C-H aliphatic and aromatic correspondently, some stretching bands were also found for $\mathrm{C}=\mathrm{C}$ at1575-1490 $\mathrm{cm}^{-1}$. In ${ }^{1} \mathrm{H}-\mathrm{NMR}$ spectra typical proton signals for $\mathrm{C}-\mathrm{H}$ aliphatic and aromatic were observed between $\delta$ 2.36-3.68, and $\delta$ 8.06-6.30 respectively.

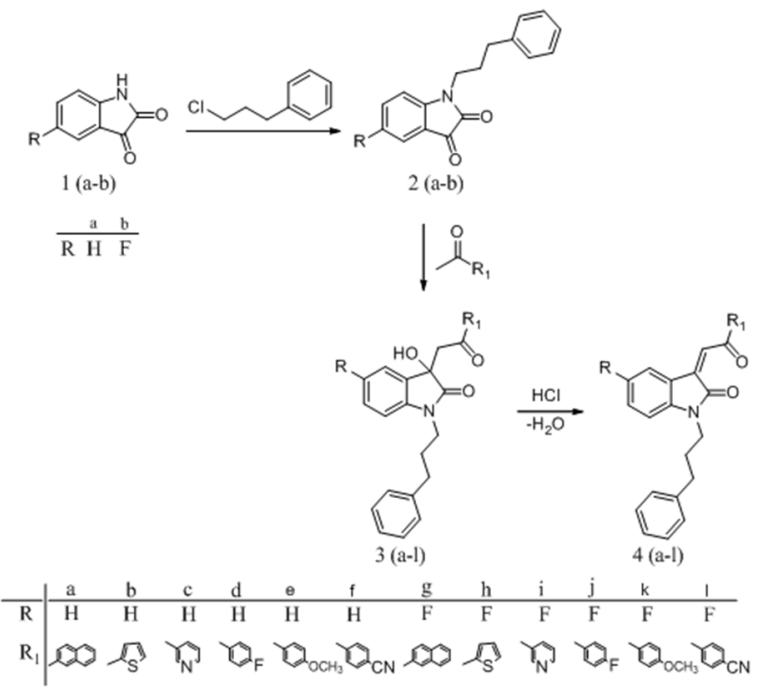

Scheme 1: Synthesis of compounds 3 (a-l) and 4 (a-l) 
Table 2: Antibacterial activity, size of inhibition zone $(\mathrm{mm})$ formed at concentration $1000 \mu \mathrm{g} / \mathrm{ml}$ of synthesized compounds $4(\mathrm{a}-\mathrm{l})$

\begin{tabular}{|c|c|c|c|c|c|c|}
\hline \multicolumn{7}{|c|}{ Zone of inhibition in $\mathrm{mm}$} \\
\hline \multirow[t]{3}{*}{ Compound } & \multicolumn{4}{|c|}{ Antibacterial activity } & \multicolumn{2}{|c|}{ Antifungal activity } \\
\hline & \multicolumn{2}{|c|}{ Gram positive } & \multicolumn{4}{|c|}{ Gram negative } \\
\hline & $\begin{array}{l}\text { Bacillus } \\
\text { subtilis }\end{array}$ & $\begin{array}{l}\text { Staphylococcus } \\
\text { aureus }\end{array}$ & $\begin{array}{l}\text { Escherichia } \\
\text { coli }\end{array}$ & $\begin{array}{l}\text { Salmonella } \\
\text { typhi }\end{array}$ & $\begin{array}{l}\text { Aspergillus } \\
\text { fumigates }\end{array}$ & $\begin{array}{l}\text { Aspergillus } \\
\text { niger }\end{array}$ \\
\hline $4 \mathrm{a}$ & $32 \pm .47$ & $31.5 \pm .23$ & $31.8 \pm .13$ & $34.5 \pm .23$ & $30.8 \pm .38$ & $25.6 \pm .24$ \\
\hline $4 \mathrm{~b}$ & $31.6 \pm .27$ & $32.96 \pm .12$ & $30.66 \pm .27$ & $33 \pm .27$ & $31 \pm .471$ & $22.2 \pm .34$ \\
\hline $4 c$ & $22 \pm .47$ & $28.6 \pm .27$ & ---------- & $28.77 \pm .32$ & $32.53 \pm .24$ & $30.76 \pm .32$ \\
\hline $4 d$ & $20.67 \pm .54$ & ---------- & $22.83 \pm .13$ & $20.33 \pm .272$ & $33.4 \pm .169$ & $29.33 \pm .27$ \\
\hline $4 \mathrm{e}$ & $34.6 \pm .27$ & $32.56 \pm .24$ & $33.66 \pm .272$ & $33.5 \pm .23$ & $34.9 \pm .42$ & $27.66 \pm .45$ \\
\hline $4 \mathrm{f}$ & $32 \pm .47$ & $28.2 \pm .16$ & $25.26 \pm .15$ & $25.73 \pm .30$ & $32.33 \pm .27$ & $28.66 \pm .27$ \\
\hline $4 \mathrm{~g}$ & $24.6 \pm .27$ & $22.8 \pm .13$ & $20.66 \pm .27$ & --------- & ---------- & $21.83 \pm .36$ \\
\hline $4 \mathrm{~h}$ & $21.33 \pm .27$ & $21.7 \pm .32$ & $21.33 \pm .27$ & --------- & $22.5 \pm .235$ & $19.6 \pm .25$ \\
\hline $4 \mathrm{i}$ & $19 \pm .47$ & $21.33 \pm .27$ & --------- & ---------- & $29.5 \pm .235$ & $22.33 \pm .27$ \\
\hline $4 j$ & $18.33 \pm .27$ & $19.5 \pm .23$ & ----------- & ----------- & $33.86 \pm .38$ & $21.56 \pm .24$ \\
\hline $4 \mathrm{k}$ & $19.34 \pm .47$ & $21.6 \pm .25$ & $15.5 \pm .23$ & -------- & $31.63 \pm .25$ & $27 \pm .47$ \\
\hline 41 & $17.66 \pm .28$ & $24.5 \pm .24$ & $19.8 \pm .36$ & $17 \pm .09$ & $26.76 \pm .32$ & $24.76 \pm .32$ \\
\hline Ciproflaxcin & 40 & 34 & 37 & 39 & & \\
\hline Amphotericin & & & & & 40 & 38 \\
\hline
\end{tabular}

No activity =----------, Note: Values are expressed in mean \pm SD $(n=3)$

\section{Antimicrobial activity}

The results of antimicrobial activities revealed that the synthesized compounds having $\mathrm{H}$ in the $5^{\text {th }}$ position of isatin ring and compounds with aromatic substitution at the $\mathrm{R}_{1}$ have shown good activity when compared with the synthesized compounds having $\mathrm{F}$ at the $5^{\text {th }}$ position. Moreover, when the aromatic ring has electronegative atom either in the ring or as a substituent, such compounds were found to be less active. Highest activity is shown by a aromatic ring with methoxy as a substituent.

All the final synthesized derivatives were taken for preliminary screening to evaluate antibacterial activity by cup plate method, in the nutrient agar medium against two gram-positive and two gramnegative bacterial strains at concentration of $1000 \mu \mathrm{g} / \mathrm{ml}$. The zone of inhibition ( $\mathrm{mm}$ ) of each derivative was ascertained and compared with Ciprofloxacin taken as standard drug for antibacterial activity. DMSO was used to prepare stock solutions of test compounds. The findings of antibacterial evaluation revealed that most of the compounds have variable activity against bacterial strains. Compounds $4 \mathrm{a}, 4 \mathrm{~b}, 4 \mathrm{e}$ and $4 \mathrm{f}$ were the active compounds which exhibited excellent activity against the bacteria in comparison to standard drug Ciprofloxacin. 4e was found to exhibit excellent activity against bacterial strains. All the final compounds were examined for antifungal activity using cup plate method, in the agar medium against two pathogenic fungal strains. The area of inhibition $(\mathrm{mm})$ of each derivative was ascertained and compared with Amphotericin B standard drug. The compounds 4c, 4d, 4e, 4f and 4j were found to be active against the fungal strains used. All the synthesized products were found to be active against $A$. fumigatus than A. Niger. However none of the compounds exhibited zone of inhibition more than that of standard.

\section{Antioxidant activity}

\section{1, 1-Diphenyl-2-picryl hydrazyl (DPPH) radical scavenging activity (RSA)}

In vitro method of scavenging of the stable DPPH radical is extensively used to evaluate the antioxidant activity in less time than other methods. DPPH is a stable free radical that can accept hydrogen radical or an electron and must thus be converted to a stable diamagnetic molecule. DPPH has an odd electron and so has a strong absorption band at $517 \mathrm{~nm}$. When this electron becomes paired off, the absorption decreases stoichiometrically with respect to the number of electrons or hydrogen atoms taken up. The DPPH antioxidant assay measures the hydrogen donating capacity of the molecules under study. When the free-radical DPPH is reduced by the sample, its colour changes from violet to yellow. Based on the structure activity relationship, it is indicated that the presence of substitution at the $5^{\text {th }}$ position of isatin ring and on the side chain influences the antioxidant potency of the molecule. Halogen substitution at position 5 of isatin ring exhibited good antioxidant activity and aromatic substitution at $\mathrm{R}_{1}$ with electronegative atom in the ring or as a substituent also showed good activity. Compounds with $\mathrm{H}$ substitution on isatin ring were found to exhibit moderate antioxidant activity. Among the synthesized compounds 4c, 4d, 4i and $4 \mathrm{j}$ were found to be more potent. Results are given in fig. 1.

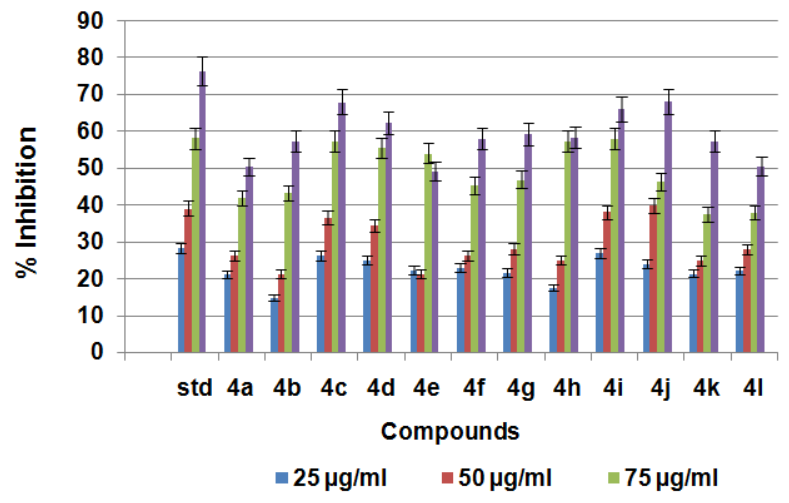

Fig. 1: DPPH radical scavenging activity of synthesized compounds at Conc. 25, 50, 75, $100 \mu \mathrm{g} / \mathrm{ml}$, The graph represents the mean $\pm S E M,(n=3), P<0.01$-significant compared to the standard group

\section{CONCLUSION}

A series of twelve compounds novel 5-substituted $N$-phenylpropyl3-substituted indoline-2-one derivatives 4(a-l) was prepared and characterized by TLC, M. P, spectral and analytical data. All the synthesized compounds were evaluated for in vitro antimicrobial activity and antioxidant activity against different bacterial and fungal strains. Compounds $4 \mathrm{a}, 4 \mathrm{~b}, 4 \mathrm{e}$ and $4 \mathrm{f}$ were highly active against gram positive and gram negative bacteria, $4 \mathrm{e}$ is found to be more potent against all the bacterial strains. Compounds $4 \mathrm{c}, 4 \mathrm{~d}, 4 \mathrm{e}$, $4 \mathrm{f}, 4 \mathrm{j}$ and $4 \mathrm{j}$ exhibited potent antifungal activity and $4 \mathrm{c}, 4 \mathrm{~d}, 4 \mathrm{i}$ and $4 \mathrm{j}$ exhibited good antioxidant activity. All the experiments were found in triplicate and the mean were calculated.

\section{AUTHORS CONTRIBUTION}

All the authors have contributed in various degrees to commencement, design, acquisition of data, analysis, interpretation of data and writing present article. 


\section{ACKNOWLEDGEMENT}

The authors are thankful to the Chairman, Department of Chemistry, Gulbarga University, Kalburgi for providing laboratory facilities to carry out the study. The author are also thankful to the Director, Central University, Hyderabad for providing spectral data. Also thankful for BioGenics Research and Training Centre in Biotechnology, Hubli for biological studies.

\section{CONFLICT OF INTERESTS}

\section{Declared none}

\section{REFERENCES}

1. Vendemiale G, Grattagliano I, Altomare E. An update on the role of free radicals and antioxidant defense in human disease. Int J Clin Lab Res 1999;29:49-55.

2. Valko M, Rhodes CJ, Moncol J, Izakovic M, Mazur M. Free radicals, metals and antioxidants in oxidative stress-induced cancer. Chem Biol Interact 2006;160:1-40.

3. Archana D, Dixitha M, Santhy Ks. Antioxidant and anti clastogenic potential of piper longum L. Int J Appl Pharm 2015;7:11-4.

4. Richardson JS. Free radicals in the genesis of Alzheimer's disease. Ann N Y Acad Sci 2006;695:73-6.

5. Olanow CW. An introduction to the free radical hypothesis in Parkinson's disease. Ann Neurol 1992;32:2-9.

6. Bankson DD, Kestin M, Rifai N. Role of free radicals in cancer and atherosclerosis. Clin Lab Med 1993;13:463-80.

7. Zelika Mega Ramadhania, Muhamad Insanu, Neni Sri Gunarti, Komar Ruslan Wirasutisna, Sukrasno Sukrasno, Rika Hartati. Antioxidant activity from ten species of myrtaceae. Asian J Pharm Clin Res 2017;Special Issue:1-7.

8. Mak JC, Chan YMM. Reactive oxidant species in asthma. Curr Opin Pulm Med 2006;12:7-11.

9. Vineet Mehta, Arun Sharma, Pallavi Kailkhura, Udayabanu Malairaman. Antioxidant, anti-inflammatory, and antidiabetic activity of hydroalcoholic extract of ocimum sanctum: an in vitro and in silico study. Asian J Pharm Clin Res 2016;9:44-9.

10. Glover V, Halket JM, Watkins PJ, Clow A, Goodwin BL, Sandler $M$. Isatin: Identity with the purified endogenous monoamine oxidase inhibitor tribulin. J Neurochem 1988;51:656-9.

11. Joaquim FM da Silva, Simon J Garden, Angelo C. The chemistry of istains: a review from 1975 to 1999. J Braz Chem Soc 2001;12:273-324.

12. Chiyanzu I, Clarkson C, Smith PJ, Lehman J, Gut J, Rosenthal PJ, et al. Design, synthesis and anti-plasmodial evaluation in vitro of new 4-aminoquinoline isatin derivatives. Bioorg Med Chem 2005;13:3249-61.

13. Da Silva JFM, Garden SJ, Pinto AC. The chemistry of istains: a review from 1975 to 1999. J Braz Chem Soc 2001;12:273-324.

14. Batanero B, Barba F. Electrosynthesis of tryptanthrin. Tetrahedron Lett 2006;47:8201-3.
15. Tarek Aboul Fadl, Fayzah AS Bin Jubair, Omima Aboul Wafa. Schiff bases of indoline-2,3-dione (isatin) derivatives and nalidixic acid carbohydrazide, synthesis, antitubercular activity and pharmacophoric model building. Eur J Med Chem 2010;45:4578-86.

16. Domenech A, Domenech-Carbo MT, Sanchez del Rio M, Vazquez de Agredos Pascual ML, Lima E. Maya blue as a nanostructured polyfunctional hybrid organic-inorganic material: the need to change paradigms. New J Chem 2009;33:2371-9.

17. Renu Dixit, AK Halve, CP Shinde, Pradeep K Soni. Synthetic and antimicrobial activity of 4 thiazolidinones. Int J Curr Pharm Res 2015;7:92-4.

18. Amal Raj A, Raghunathan R, Sridevi Kumari MR, Raman N. Synthesis, antimicrobial and antifungal activity of a new class of spiro pyrrolidines. Bioorg Med Chem 2003;11:407-19.

19. Quenelle D, Keith K, Kern E. In vitro and in vivo evaluation of isatin- $\beta$-thiosemicarbazone and marboran against vaccinia and cowpox virus infections. Antiviral Res 2006;71:24-30.

20. Bal TR, Anand B, Yogeeswari P, Sriram D. Synthesis and evaluation of anti-HIV activity of isatin $\beta$-thiosemicarbazone derivatives. Bioorg Med Chem Lett 2005; 15:4451-5.

21. Karal N, Gürsoy A, Kandemirli F, Shvets N, Kaynak FB, Ozbey S, et al. Synthesis and structure-antituberculosis activity relationship of $1 \mathrm{H}$-indole-2,3-dione derivatives. Bioorg Med Chem 2007;15:5888-904.

22. Gursoy A, Karal N. Synthesis and primary cytotoxicity evaluation of 3-[[(3-phenyl-4(3H)-quinazolinone-2-yl) mercaptoacetyl]hydrazono]-1H-2-indolinones. Eur J Med Chem 2003;38:633-43.

23. Sridhar SK, Ramesh A. Synthesis and pharmacological activities of hydrazones, Schiff and Mannich bases of isatin derivatives. Biol Pharm Bull 2001;24:1149-52.

24. Verma M, Pandeya SN, Singh KN, Stables JP. Anticonvulsant activity of schiff bases of isatin derivatives. Acta Pharm 2004;54:49-56.

25. Bhattacharya SK. Anticonvulsant activity of intraventricularly administered atrial natriuretic peptide and its inhibition by isatin. Biog Amines 1988;14:131-41.

26. Gang Chen, Ye Wang, Xiaojiang Hao, Shuzhen Mu, Qianyun Sun. Simple isatin derivatives as free radical scavengers: synthesis, biological evaluation and structure-activity relationship. Chem Cent J 2011;37:1-5.

27. Basavaraj Shivabasappa Naraboli, Jayaprakash Sharanappa Biradar. Synthesis, characterization and biological evaluation of indole derivatives bearing benzimidazole/benzothiazole moiety. Int J Pharm Pharm Sci 2017;9:128-38.

\section{How to cite this article}

- Pushpa, Basavaraj S Naraboli, JS Biradar. Synthesis, characterization, and biological activity of novel N-phenylpropyl3-substituted indoline-2-one derivatives. Int J Pharm Pharm Sci 2017;9(10):165-170. 